\title{
Osteoarthritis of the foot: a review of the current state of knowledge
}

\author{
Annamaria Iagnocco ${ }^{1}$, Chiara Rizzo ${ }^{1}$, Angelica Gattamelata ${ }^{1}$, Caterina Vavala ${ }^{1}$, \\ Fulvia Ceccarelli ${ }^{1}$, Elena Cravotto ${ }^{1}$, Guido Valesini ${ }^{1}$
}

${ }^{1}$ Rheumatology Unit, Dipartimento di Medicina Interna e Specialità Mediche, Sapienza Università di Roma, Rome, Italy

\begin{abstract}
Foot osteoarthritis (OA) is a very common disease that mainly affects older people deeply influencing their quality of life. The joint most frequently involved in the articular degenerative process is the first metatarsophalangeal joint. Its severe impairment may lead to a specific clinical pattern known as hallux rigidus that often requires surgical treatment. Currently conventional radiograms of feet associated with an accurate clinical examination should be performed in order to diagnose foot OA. However, new imaging modalities as ultrasonography and magnetic resonance imaging are emerging as valuable tools to assess foot OA. Therapeutic options for foot OA consist of conservative strategies, including life-style modification and pharmacological treatment, options that are usually adopted in early-stage disease and in invasive surgical procedures reserved to late-stage conditions. At the present time there is a lack of evidence in international literature specifically dealing with foot $\mathrm{OA}$, so further investigation on this topic is required to clarify its pathogenesis, the diagnostic pathway and the best clinical management.
\end{abstract}

Keywords: osteoarthritis, foot, ultrasound

Osteoarthritis (OA) is a degenerative disorder that results from the biochemical breakdown of articular cartilage in the synovial joints and is the commonest cause of arthritis and a leading cause of pain and disability worldwide [1].

Biomechanical and biochemical factors, such as the production of several chemokines, cytokines, and growth factors, determine an aberrant chondrocyte activation that contributes to the disruption of articular homeostasis. The imbalance between pro- and anti-inflammatory molecules may influence the progression of cartilage damage [2].

Received 06.01.2013 Accepted 15.01.2013

Med Ultrason

2013, Vol. 15, No 1, 35-40

Corresponding author: Annamaria Iagnocco,

Dipartimento Medicina Interna e

Specialità Mediche: Reumatologia,

Sapienza Università di Roma,

V.le del Policlinico 155, Rome - 00161, Italy.

Tel: +3906 49974634, Fax: +390649974642

Email: annamaria.iagnocco@uniroma1.it
OA accounts for $15 \%$ of all musculoskeletal consultations in patients aged 45 years and over in primary care [3]. This degenerative articular disease predominantly affects the knees, hips, hands and feet. Whereas the knee, the hip and the hand have received considerable attention, the foot has been relatively neglected yet the first metatarsophalangeal joint is one of the joints most frequently affected by OA [4].

The aim of this review is to provide an overview of the current knowledge on foot OA, concerning risk factors, clinical features, diagnosis and treatment.

\section{Epidemiology and pathomechanism}

OA is the leading cause of chronic pain and disability among older people in developed countries. A podiatric supplement to the US National Health Interview Survey estimated that $24 \%$ of the population has at least one foot ailment, with older adults experiencing more troubles than younger adults [5]. Recent prevalence data from the Framingham Study reported that $19 \%$ of men and $29 \%$ 
of women reported foot pain on most days of the month, with prevalence of pain at specific foot locations ranging from $7 \%$ to $13 \%$ [6].

The foot is involved with a variable frequency according to the joint sites, hindfoot $\mathrm{OA}$ is quite uncommon, but forefoot $\mathrm{OA}$ is a frequent joint disease, particularly at the level of the first metatarsophalangeal joint, that represents the prime site of OA changes. It is estimated that OA of the big toe joint affects $35 \%$ to $60 \%$ of adults aged over 65 years [7].

The midfoot is rarely involved in OA alterations and only few research groups have described non-Charcot arthropathy of the midfoot, including patients with primary OA in this joint site [8-10].

The literature suggests that midfoot $\mathrm{OA}$ is usually post-traumatic. Midfoot injuries affect approximately 55,000 people per year and are commonly seen in the athletic population [11]. Despite their seemingly low incidence, they are particularly important because as many as $20 \%$ are missed or misdiagnosed [12].

Moreover OA of the tarsometatarsal joints has emerged as a challenging problem due to its high potential for foot pain and chronic secondary disability. In fact subjects with midfoot OA experience foot pain which deeply limits their participation in walking and weightbearing activities.

The etiology of foot OA is still poorly understood, in fact only a few studies in international literature have highlighted major risk factors for developing foot OA $[13,16-21]$. It has been linked to trauma, sport injuries, inflammatory arthropathy, mechanical stress and idiopathic osteoarthritis [13]. Several authors have suggested that, similarly to other forms of non-traumatic OA, foot $\mathrm{OA}$ may be a result of abnormally high mechanical stress due to abnormal structural factors $[14,15]$. Davitt et al. hypothesized that aberrant loading may be the result of toe length abnormalities, as patients with idiopathic midfoot OA were more likely to have a second toe longer than the great toe [16] . OA of the first metatarsophalangeal joint of the foot, commonly referred to as hallux limitus or hallux rigidus, is thought to be caused by the compression of the dorsal aspect of the joint during the propulsive phase of gait in people with an excessively wide first metatarsal, wide proximal phalanx and long sesamoids. Furthermore people with flat feet or high arches are at greater risk for developing foot OA. A recent research has indicated that older people with radio-graphically-confirmed $\mathrm{OA}$ of the talonavicular joint and navicular-first cuneiform joint have significantly flatter feet in association with a significant greater dynamic loading of the midfoot when walking [17-20]. Finally, Yu et al suggested that the aberrant loading may be a result of past shoe use or footwear, noting that chronic high-heel use increased the risk of midfoot OA [21].

In addition, general factors as aging and obesity seem to play a key role in the pathomechanism of OA. Aging determines the reduction of cartilage volume, proteoglycan content, cartilage vascularization, and cartilage perfusion leading to favorable conditions to initiate the OA process. Obesity increases the mechanical stress in weight-bearing joints, so it particularly affects lower limb joints. Furthermore it has been demonstrated that obese patients alter their movement strategies of every day movements causing important changes in joint biomechanics. Both factors contribute to the degenerative process that involves joint cartilage [22-24].

\section{Clinical features}

The key feature of OA is the progressive destruction of joint cartilage that causes reduction of articular space, subcondral sclerosis, subcondral cysts, synovial phlogosis and osteophyte formation.

The usual symptoms associated with foot OA include: pain and stiffness of the affected foot, swelling near the affected joint, limited range of motion, difficulty in walking and bony protrusions. There are 28 bones and more than 30 joints in the human foot. The foot joints that are most commonly affected by osteoarthritis include:

- the 3 joints of the hindfoot (talocalcaneal joint, talonavicular joint, calcaneocuboid joint)

- the midfoot (metatarsocuneiform joint)

- the great toe (first metatarsophalangeal joint)

OA releated pain diminishes the quality of life and limits physical functions. Patients have problem in doing housework as well as leisure activities $[25,26]$. In addition severe OA leads to deformities that typically affect the metatarsophalangeal joint causing significant limitation of motion and function of feet [27]. Structural alterations of the first metatarsophalangeal joint are described as either hallux limitus or hallux rigidus. The term used depends on the magnitude of available joint motion and the severity of joint degeneration. Hallux limitus is characterized by restricted sagittal plane motion (primarily dorsiflexion) caused by periarticular osteophytes; whereas hallux rigidus displays an absence of joint motion due to end stage degenerative joint disease and subsequent joint ankylosis. Although the terms are often used interchangeably, hallux rigidus has been adopted by the World Health Organization in the International Classification of Diseases [28,29].

Foot joints can even be affected by a rare form of OA that is characterized by erosions. Erosive OA has a poor clinical outcome. Patients experience several inflamma- 
tory episodes due to cartilage debris that activate the inflammatory process resulting in progressive joint damage and structural change [30].

\section{Diagnosis}

Physical examination can detect swelling, bone spurs or other deformities, limited range of motion and pain which occurs with movement. However, to define the diagnosis conventional radiographs are required to assess typical alterations found in foot $\mathrm{OA}$ joints.

Different radiographic scales to identify and quantify foot OA exist, but the most frequently used is the one elaborated by Kellgren and Lawrence. This scale ranges from 0 to 4: 0 corresponding to absence of $\mathrm{OA}$ features and 4 to severe $\mathrm{OA}$.

The radiological features usually examined are:

1) The formation of osteophytes on the joint margins or, in the case of the knee joint, on the tibial spines.

2) Periarticular ossicles; in particular at the level of distal and proximal interphalangeal joints.

3) Narrowing of joint cartilage associated with sclerosis of subchondral bone.

4) Small pseudocystic areas with sclerotic walls situated usually in the subchondral bone.

5) Altered shape of the bone ends, particularly in the head of femur [31].

In $2007 \mathrm{Menz}$ at al [32] presented an atlas of radiographic features of OA in commonly affected joints of the foot based on observations of osteophytes and joint space narrowing. The aim was to standardize the documentation and interpretation of foot $\mathrm{OA}$ for epidemiological studies and clinical trials. Authors suggested a foot radiography protocol to identify OA. According to them weight-bearing dorso-plantar and lateral radiographic projections have to be performed in order to document osteophytes and joint space narrowing from both, in fact it was evident that the dorso-plantar projection provided the greatest clarity for some joints, whereas the lateral projection was the most suitable for others. The atlas was demonstrated to be acceptably reliable, and considered useful to document features in individual joints or to calculate a global foot OA score [32].

Recently many researchers have described the importance of ultrasonography (US) in evaluating joints and periarticular structures [33]. In the past decades the impressing technological development of US machines has encouraged the application of US in rheumatological clinical practice as a sensitive and bedside imaging technique. It has even been included in the classification criteria for polymyalgia rheumatica in 2012 [33-35]. US, differently from radiography, can detect inflammation, cartilage reduction and even early erosions. The existing literature dealing with the ultrasonographic assessment of foot has focused on several rheumatic diseases as gout, early arthritis, rheumatoid arthritis and psoriatic arthritis [36-40]. However, even if US has been depicted as a valuable tool to assess the severity of osteoarthritic lesions and to accurately monitor their eventual progression, currently there is only one article that deals with the application of US in foot OA [41]. In this study bilateral US of the midfoot and forefoot joints was performed assessing both inflammatory (joint effusion, synovial hypertrophy, local pathologic vascularization at Power Doppler, big toe bursitis) and structural (osteophytes, metatarsophalangeal joints subluxation) abnormalities. Authors concluded that US was a useful imaging technique for analysing both inflammatory and structural damage lesions at foot joints level in OA. In addition, it was demonstrated to be more sensitive than clinical examination in the detection of inflammatory abnormalities [42].

Finally, clinicians should consider magnetic resonance imaging (MRI) too. MRI-based research is becoming increasingly more common in the OA research community. Novel MRI-based semiquantitative scoring systems have been proposed for the knee, hip and hand OA. MRI identifies earlier changes in OA joints compared to standard radiography. A systematic review published in 2011 examined the validity of MRI in OA and authors concluded that MRI is the current most valuable imaging tool for the assessment of OA due to its unique ability to visualize multiple individual tissue pathologies relevant in regard to pain and other clinical outcomes. In fact, MRI is able to detect bone marrow lesions, synovitis and effusion that have been related to pain. On the basis of these results, MRI is now recommended for clinical trials in terms of cartilage morphology assessment [4345].

Recent studies have focused their attention on the delayed gadolinium-enhanced magnetic resonance imaging of cartilage (dGEMRIC). Thanks to this particular technique it is possible to estimate the cartilage glycosaminoglican (GAG) content. It seems to be a promising and sensitive biomarker to reveal degenerative modifications in cartilage quality in the very early stages of OA [46].

\section{Treatment}

Therapeutic strategies for foot OA are based on a wide spectrum of options and need to be always patient oriented. Treatment decisions should be made according to a staged approach. Conservative treatment should be advised in early OA while more invasive procedures 
should be considered when symptoms and deformities become unbearable and deeply affect the patient's quality of life.

\section{Conservative therapy}

Weight-loss is often proposed as an important endpoint to reduce foot pain. However only few studies have investigated the role of weight loss in improving foot $\mathrm{OA}$ related pain, so current evidence dealing with this topic is still too limited [22].

Exercise has been proven to be a protective factor against $\mathrm{OA}$ as it stimulates biosynthetic activity of chondrocytes. However, excessive physical exercise can damage the articular and periarticular structures. So, patients with OA capable of exercise should be encouraged to partake in a low-impact aerobic exercise program (walking, biking, swimming or other aquatic exercise) $[47,48]$.

Furthermore, two studies in literature have demonstrated how orthotic treatment for midfoot OA significantly decreases pain levels, increases activity levels and footwear comfort $[49,50]$.

As pharmacological options, chondroprotective agents and analgesics should be used.

Different studies have evaluated in randomized controlled trials and systematic reviews the effect of glucosamine, chondroitin sulfate and collagen derivatives in blocking the progression of cartilage denaturation in OA. The results showed a global beneficial trend even if compared with placebo, glucosamine, chondroitin, and their combination did not significantly reduce joint pain or have an impact on the narrowing of joint space [51,52].

The analgesic therapy recommended for the initial management of patients with OA includes acetaminophen, oral and topical non-steroidal anti-inflammatory drugs, tramadol, and intra-articular corticosteroid injections; while intra-articular hyaluronate injections, duloxetine, and opioids are conditionally recommended in patients who have an inadequate response to initial therapy. Opioid analgesics are strongly recommended in patients who cannot undergo surgical treatment, as total joint arthroplasty, after having failed medical therapy [53].

Recently there has been an important increase of interest in intra-articular application of hyaluronic acid to treat OA joints. Hyaluronic acid provides viscosupplementation in order to restore the viscosity and elasticity of synovial fluid. Its efficacy has been demonstrated in knee, hip and ankle OA. There is a lack of data concerning hyaluronic intra-articular injections on foot joints [54-56].

\section{Surgery}

In cases of severe deforming foot OA, surgery should be considered as an endpoint therapeutic approach. In particular the international research is focused in the treatment of OA of the big toe. A systematic review published in 2010 described surgical options to treat hallux rigidus and/or limitus. Procedures include cheilectomy (removal of a bony lump at the joint margin), arthrodesis (surgical fusion of bones), arthroplasty (joint replacement via remodelling or implant), osteotomy (cutting bone to shorten, lengthen or realign), soft tissue release and sesamoid release or excision [57-59].

\section{Conclusions}

Foot OA is a common musculoskeletal disorder that particularly affects older people worsening their quality of life. The joint most frequently involved in the degenerative progression of OA is the first metatarsophalangeal one. The pathogenesis and risk factors for foot OA need to be further investigated. US and MRI are gaining importance in the diagnostic pathway of OA, even if conventional radiology must still be referred as the "gold standard" imaging technique. Therapeutic strategy for foot $\mathrm{OA}$, involving conservative and invasive treatments, should always be related to the OA stage in the single patient. New researches dealing with the treatment of foot $\mathrm{OA}$ are required in order to make the best decisions for the management of this articular degenerative disease.

\section{Conflict of interest: none}

\section{References}

1. Arden N, Nevitt MC. Osteoarthritis: epidemiology. Best Pract Res Clin Rheumatol 2006; 20: 3-25.

2. Dieppe PA. Osteoarthritis and related disorders. Introduction and history. In: Klippel JH, Dieppe PA (Eds.). Rheumatology. Mosby, London 1998: 8.1.1-8.1.2

3. National Collaborating Centre for Chronic Conditions. Osteoarthritis: National Clinical Guideline for Care and Management in Adults. London: Royal College of Physicians; 2008.

4. Van Saase JL, Vandenbroucke JP, Van Romunde LK, Valkenburg HA. Osteoarthritis and obesity in the general population. A relationship calling for an explanation. J Rheumatol 1988; 15: 1152-1158.

5. Greenberg L, Davis H. Foot problems in the US. The 1990 National Health Interview Survey. J Am Podiatr Med Assoc 1993; 83: 475-483.

6. Dufour AB, Broe KE, Nguyen US, et al. Foot pain: is current or past shoewear a factor? Arthritis Rheum 2009; 61: 1352-1358.

7. Van Saase JL, Van Romunde LK, Cats A, Vandenbroucke JP, Valkenburg HA. Epidemiology of osteoarthritis: Zoetermeer survey. Comparison of radiological osteoarthritis in 
a Dutch population with that in 10 other populations. Ann Rheum Dis 1989; 48: 271-280.

8. Kuo RS, Tejwani NC, Digiovanni CW, et al. Outcome after open reduction and internal fixation of Lisfranc joint injuries. J Bone Joint Surg Am 2000;82:1609-1618.

9. Horton GA, Olney BW. Deformity correction and arthrodesis of the midfoot with a medial plate. Foot Ankle 1993; 14: 493-499.

10. Johnson JE, Johnson KA. Dowel arthrodesis for degenerative arthritis of the tarsometatarsal (Lisfranc) joints. Foot Ankle 1986; 6: 243-253.

11. Curtis MJ, Myerson M, Szura B. Tarsometatarsal joint injuries in the athlete. Am J Sports Med 1993; 21: 497-502.

12. Goossens M, De Stoop N. Lisfranc's fracture-dislocations: etiology, radiology, and results of treatment. A review of 20 cases. Clin Orthop Relat Res 1983; 176: 154-162.

13. Riskowski J, Dufour AB, Hannan MT. Arthritis, foot pain and shoe wear: current musculoskeletal research on feet. Curr Opin Rheumatol 2011;23:148-155.

14. Englund M. The role of biomechanics in the initiation and progression of OA of the knee. Best Pract Res Clin Rheumatol 2010; 24: 39-46.

15. Adatia A, Rainsford KD, Kean WF. Osteoarthritis of the knee and hip. Part I: aetiology and pathogenesis as a basis for pharmacotherapy. J Pharm Pharmacol 2012; 64: 617625.

16. Davitt JS, Kadel N, Sangeorzan BJ, Hansen ST Jr, Holt SK, Donaldson-Fletcher E. An association between functional second metatarsal length and midfoot arthritis. J Bone Joint Surg Am 2005; 87: 795-800.

17. Roth RD. Talonavicular joint osteoarthritis (osteoarthrosis). J Am Podiatry Assoc 1982; 72: 237-243.

18. Sayeed SA, Khan FA, Turner NS 3rd, Kitaoka HB. Midfoot arthritis. Am J Orthop 2008; 37: 251-256.

19. Zammit GV, Menz HB, Munteanu SE. Structural factors associated with hallux limitus/rigidus: a systematic review of case control studies. J Orthop Sports Phys Ther 2009; 39: 733-742.

20. Davitt JS, Kadel N, Sangeorzan BJ, Hansen ST Jr, Holt SK, Donaldson-Fletcher E. An association between functional second metatarsal length and midfoot arthritis. J Bone Joint Surg Am 2005; 87: 795-800.

21. Yu J, Cheung JT, Fan Y, Zhang Y, Leung AK, Zhang M. Development of a finite element model of female foot for high-heeled shoe design. Clin Biomech 2008; 23 Suppl 1: S31-38.

22. Butterworth PA, Landorf KB, Smith SE, Menz HB. The association between body mass index and musculoskeletal foot disorders: a systematic review. Obes Rev 2012; 13: 630-642.

23. Wearing SC, Hennig EM, Byrne NM, Steele JR, Hills AP. Musculoskeletal disorders associated with obesity: a biomechanical perspective. Obes Rev 2006; 7: 239-250.

24. Runhaar J, Koes BW, Clockaerts S, Bierma-Zeinstra SM. A systematic review on changed biomechanics of lower extremities in obese individuals: a possible role in development of osteoarthritis. Obes Rev 2011; 12: 1071-1082.
25. Golightly YM, Hannan MT, Shi XA, Helmick CG, Renner JB, Jordan JM. Foot symptoms are independently associated with poor self-reported and performance-based physical function: The Johnston County osteoarthritis project. Arthritis Care Res 2011; 63: 654-659.

26. Bergin SM, Munteanu SE, Zammit GV, Nikolopoulos N, Menz HB. Impact of first metatarsophalangeal joint osteoarthritis on health-related quality of life. Arthritis Care Res 2012; 64: 1691-1698.

27. McMaster MJ. The pathogenesis of hallux rigidus. J Bone Joint Surg 1978;60:82-87.

28. Camasta CA. Hallux limitus and hallux rigidus. Clinical examination, radiographic findings, and natural history. Clin Podiatr Med Surg 1996;13:425-428.

29. World Health Organization: International Statistical Classification of Diseases and Related Health Problems, 10th Revision; 2007.

30. Anandaraja AP, Thiele RG, Monu J. Erosive ostoarthritis is a progressive disease that commonly affects the feet. Arthritis Rheum 2009; 60: S71.

31. Kellgren JH, Lawrence JS. Radiological assessment of osteo-arthrosis. Ann Rheum Dis 1957; 16: 494-501.

32. Menz HB, Munteanu SE, Landorf KB, Zammit GV, Cicuttini FM. Radiographic classification of osteoarthritis in commonly affected joints of the foot. Osteoarthritis Cartilage 2007; 15: 1333-1338.

33. Filippucci E, Iagnocco A, Meenagh G, et al. Ultrasound imaging for the rheumatologist. Clin Exp Rheumatol 2006;24:1-5.

34. Dasgupta B, Cimmino MA, Kremers HM, et al. 2012 Provisional classification criteria for polymyalgia rheumatica: a European League Against Rheumatism/American College of Rheumatology collaborative initiative. Arthritis Rheum 2012; 64: 943-954

35. Dasgupta B, Cimmino MA, Maradit-Kremers H, et al. 2012 provisional classification criteria for polymyalgia rheumatica: a European League Against Rheumatism/American College of Rheumatology collaborative initiative. Ann Rheum Dis 2012; 71: 484-492.

36. Filippucci E, Meenagh G, Delle Sedie A, et al. Ultrasound imaging for the rheumatologist XXXVI. Sonographic assessment of the foot in gout patients. Clin Exp Rheumatol 2011; 29: 901-905.

37. Meenagh G, Sakellariou G, Iagnocco A, et al. Ultrasound imaging for the rheumatologist XXXIV. Sonographic assessment of the painful foot. Clin Exp Rheumatol 2011; 29: 601-603.

38. Scirè CA, Iagnocco A, Meenagh G, et al. Ultrasound imaging for the rheumatologist XXXIII. Sonographic assessment of the foot in early arthritis patients. Clin Exp Rheumatol 2011; 29: 465-469.

39. Riente L, Delle Sedie A, Scirè CA, et al. Ultrasound imaging for the rheumatologist. XXXI. Sonographic assessment of the foot in patients with rheumatoid arthritis. Clin Exp Rheumatol 2011; 29: 1-5.

40. Delle Sedie A, Riente L, Filippucci E, et al. Ultrasound imaging for the rheumatologist XXXII. Sonographic assess- 
Osteoarthritis of the foot: a review of the current state of knowledge

ment of the foot in patients with psoriatic arthritis. Clin Exp Rheumatol 2011; 29: 217-222.

41. Iagnocco A. Imaging the joint in osteoarthritis: a place for ultrasound? Best Pract Res Clin Rheumatol 2010; 24: 27 38 .

42. Iagnocco A, Filippucci E, Riente L, et al. Ultrasound imaging for the rheumatologist XXXV. Sonographic assessment of the foot in patients with osteoarthritis. Clin Exp Rheumatol 2011; 29: 757-762.

43. Hunter DJ, Zhang W, Conaghan PG, et al. Systematic review of the concurrent and predictive val-idity of MRI biomarkers in OA. Osteoarthritis Cartilage 2011; 19: 557588.

44. Conaghan PG, Hunter DJ, Maillefert JF, Reichmann WM, Losina E. Summary and recommendations of the OARSI FDA osteoarthritis Assessment of Structural Change Working Group. Osteoarthritis Cartilage 2011; 19: 606-610.

45. Hayashiy D, Roemer FW, Guermazi A. Osteoarthritis year 2011 in review: imaging in OA-a radiologists' perspective. Osteoarthritis Cartilage 2012; 20: 207-214.

46. Dahlberg LE, Lammentausta E, Tiderius CJ, et al. In vivo monitoring of joint cartilage- lessons to be learned by delayed gadolinium enhanced magnetic resonance imaging of cartilage. European Musculoskeletal Review 2012; 7: 5862.

47. Roddy E, Zhang W, Doherty M. Aerobic walking or strengthening exercise for osteoarthritis of the knee? A systematic review. Ann Rheum Dis 2005; 64: 544-548.

48. Hunter DJ, Eckstein F. Exercise and osteoarthritis. J Anat 2009; 214: 197-207.

49. Rao S, Baumhauer JF, Becica L, Nawoczenski DA. Shoe inserts alter plantar loading and function in patients with midfoot arthritis. J Orthop Sports Phys Ther 2009; 39: 522531.

50. Ibuki A, Cornoiu A, Clarke A, Unglik R, Beischer A. The effect of orthotic treatment on midfoot osteoarthritis as- sessed using specifically designed patient evaluation questionnaires. Prosthet Orthot Int 2010; 34: 461-471.

51. Wandel S, Juni P, Tendal B, et al. Effects of glucosamine, chondroitin, or placebo in patients with osteoarthritis of hip or knee: network meta-analysis. BMJ 2010; 341: c4675.

52. Van Vijven PJ, Luijsterburg PA, Verhagen AP, van Osch GJ, Kloppenburg M, Bierma-Zeinstra SM. Symptomatic and chondroprotective treatment with collagen derivatives in osteoarthritis: a systematic review. Osteoarthritis Cartilage 2012; 20: 809-821.

53. Hochberg MC, Altman RD, April KT, et al; American College of Rheumatology. American College of Rheumatology 2012 recommendations for the use of non-pharmacologic and pharmacologic therapies in osteoarthritis of the hand, hip, and knee. Arthritis Care Res 2012; 64: 465-474.

54. Grogan KA, Chang TJ, Salk RS. Update on viscosupplementation in the treatment of osteoarthritis of the foot and ankle. Clin Podiatr Med Surg 2009; 26: 199-204.

55. Munteanu SE, Zammit GV, Menz HB, et al. Effectiveness of intra-articular hyaluronan (Synvisc, hylan G-F 20) for the treatment of first metatarsophalangeal joint osteoarthritis: a randomised placebo-controlled trial. Ann Rheum Dis 2011; 70: 1838-1841.

56. Pons M, Alvarez F, Solana J, Viladot R, Varela L. Sodium hyaluronate in the treatment of hallux rigidus. A singleblind, randomized study. Foot Ankle Int 2007; 28: 38-42.

57. Becher C, Kilger R, Thermann H. Results of cheilectomy and additional microfracture technique for the treatment of hallux rigidus. Foot Ankle Surg 2005; 3: 155-160.

58. Gibson JN, Thomson CE. Arthrodesis or total replacement arthroplasty for hallux rigidus: A randomized controlled trial. Foot Ankle Int 2005; 26: 680-690.

59. O'Doherty DP, Lowrie IG, Magnussen PA, Gregg PJ. The management of the painful first metatarsophalangeal joint in the older patient. Arthrodesis or Keller's Arthroplasty? J Bone Joint Surg 1990; 72: 839-842. 